\title{
Lobectomy is superior to segmentectomy for peripheral high grade non-small cell lung cancer $\leq 2 \mathrm{~cm}$
}

\author{
Mirza Zain Baig', Syed S. Razi ${ }^{2}$, Joanna F. Weber ${ }^{1}$, Cliff P. Connery ${ }^{3}$, Faiz Y. Bhora ${ }^{1}$ \\ ${ }^{1}$ Division of Thoracic Surgery, Rudy L. Ruggles Biomedical Research Institute, Nuvance Health Systems, Danbury, CT, USA; ${ }^{2}$ Division of Thoracic \\ Surgery, Memorial Healthcare System, South Broward, FL, USA; ${ }^{3}$ Division of Thoracic Surgery, Nuvance Health Systems, Poughkeepsie, NY, USA \\ Contributions: (I) Conception and design: MZ Baig, SS Razi, FY Bhora; (II) Administrative support: FY Bhora, CP Connery; (III) Provision of \\ study materials or patients: MZ Baig, SS Razi, JF Weber; (IV) Collection and assembly of data: MZ Baig, SS Razi, JF Weber; (V) Data analysis and \\ interpretation: MZ Baig, SS Razi, JF Weber; (VI) Manuscript writing: All authors; (VII) Final approval of manuscript: All authors. \\ Correspondence to: Faiz Y. Bhora, MD, FACS. Division of Thoracic Surgery, Rudy L. Ruggles Biomedical Research Institute, Nuvance Health \\ Systems, 111 Osborne Street Danbury, CT 06810, USA. Email: faiz.bhora@nuvancehealth.org.
}

Background: Current practice guidelines recommend the following criteria for segmentectomy for nonsmall cell lung cancer (NSCLC): size $\leq 2 \mathrm{~cm}$, margins $\geq 2 \mathrm{~cm}$ and no lymph node involvement. We sought to further stratify the selection criteria for segmentectomy for small peripheral high-grade tumors.

Methods: This retrospective database study was conducted using the Surveillance, Epidemiology and End Results (SEER) database. We queried for patients with high-grade (poorly differentiated/undifferentiated) pathological (p)T1a/b peripheral NSCLC (tumor size $\leq 2 \mathrm{~cm}$ ), who underwent either lobectomy or segmentectomy between 2004 and 2015. Patients with node-positive disease or those who received any form of induction or adjuvant treatments were excluded.

Results: A total of 4,332 patients met the inclusion criteria, with 3,977 patients (91.8\%) treated with lobectomy and 355 patients (8.2\%) who underwent segmentectomy. In a propensity matched pair analysis of 640 patients, lobectomy ( $\mathrm{n}=320$ ) showed significantly improved 5 -year survival of $45.9 \%$ vs. $33.8 \%$ for segmentectomy $(\mathrm{n}=320), \mathrm{P}<0.01$. In a multivariate Cox regression analysis, lobectomy was associated with significantly improved survival (HR: 0.84, 95\% CI: 0.714-0.989, P=0.036). Interestingly, married status, adenocarcinoma histology, number of lymph nodes sampled were associated with better survival $(\mathrm{P}<0.05)$, while advanced age and male gender had worse survival outcomes $(\mathrm{P}<0.05)$.

Conclusions: For small peripheral NSCLC $\leq 2 \mathrm{~cm}$ and high grades of tumor differentiation, lobectomy is associated with better long-term survival outcomes as compared to segmentectomy. Additional data is needed to further stratify various NSCLC histologies with their respective grades to allow for better selection for segmentectomy.

Keywords: Lobectomy; segmentectomy; sublobar resection; high grade; non-small cell lung cancer (NSCLC)

Submitted Mar 31, 2020. Accepted for publication Jul 15, 2020.

doi: $10.21037 /$ jtd-20-1530

View this article at: http://dx.doi.org/10.21037/jtd-20-1530

\section{Introduction}

Lung cancer is the leading cause of cancer related death (1-5) with an estimated yearly global incidence of 1.8 million and an estimated yearly mortality of 1.6 million (1). Surgery is the treatment of choice for early stage non-small cell lung cancer (NSCLC) and can give five-year survival rates as high as $70 \%(3,6)$. Anatomical lobectomy with lymph node dissection is considered the standard of care for clinical (c) T1, N0 peripheral lung nodules since the landmark trial reported by the Lung Cancer Study Group (LCSG) in 1995 (2,3,5,7-10). An important prerequisite for lobectomy however, is the adequacy of pulmonary function and functional status of the patients. There are an estimated 
$30 \%$ of patients with surgically resectable tumors, but are medically unfit for lobectomy (8). Segmentectomy has traditionally been considered a compromise operation for patients with limited pulmonary reserve, advanced age, severe comorbidities or other clinical features that may preclude lobectomy $(2,7)$. However, there is a growing body of evidence that supports anatomic segmentectomy with oncologic outcomes similar to lobectomy in early stage NSCLC less than $2 \mathrm{~cm}$ in greatest dimension $(4,7)$.

The risk of developing metachronous, recurrent or second primary lung cancer has been estimated at $1 \%$ to $2 \%$ per patient-year. Hence, patients following segmentectomy are more likely to withstand further therapeutic lung cancer surgery (11-13). Nevertheless, selection criteria for lobectomy versus segmentectomy should include consideration of several important clinical variables, not fully defined yet, and caution must be exercised regarding inappropriate use of segmentectomy in patients otherwise good surgical candidates for lobectomy. Important clinical determinants that strongly support lobectomy over segmentectomy include, but not limiting to, young age, aggressive tumor histology and (c)N1/N2 disease $(14,15)$. The purpose of this study is to further characterize long term survival outcomes following lobectomy and segmentectomy in patients with high grade (poorly differentiated and undifferentiated) NCSLC $\leq 2 \mathrm{~cm}$ using Surveillance, Epidemiology and End Results (SEER) database. We present the following article in accordance with the STROBE checklist (available at http://dx.doi. org/10.21037/jtd-20-1530).

\section{Methods}

\section{Study population}

We conducted a retrospective database study using the SEER database, a registry of the National Cancer Institute (16). The SEER database is an extensive source of population-based data that covers approximately $28 \%$ of the US population. We queried 18 SEER registries (November 2018 submission) from 2004 to 2015 for all patients with high grade early stage pathological (p)T1, $\leq 2 \mathrm{~cm}$, nodenegative disease status (p)N0 NSCLC who underwent either segmentectomy or lobectomy. Though the SEER database does not include the TNM $8^{\text {th }}$ edition for tumor staging, all cases were manually staged according to the $8^{\text {th }}$ edition by incorporating data on tumor size, lymph node and metastasis status. Using the "International Classification of Disease, Third Edition" (ICD-O-3) codes, we queried for primary site "lung" (ICD-O-3 C34.1-C34.9). Tumor of the main bronchus (ICD-O-3 C34.0) overlapping tumors and tumors with unspecified locations were excluded. The SEER database uses the four-tier system for tumor grade. Based on this, we included only grade III (poorly differentiated) and grade IV (undifferentiated) tumors in our cohort. Our study conforms to the Declaration of Helsinki (as revised in 2013). Since our study utilizes de-identified data from a publicly accessible database, individual consent is waived and our study is exempt from IRB review.

\section{Selection of covariates}

Data extracted from SEER included patient demographics (age, sex, marital status), tumor characteristics (location, histology, grade, size), surgery type (lobectomy $v s$. segmentectomy), number of lymph nodes sampled, overall survival (OS) in months, cancer specific survival in months and patient status at the end of the follow-up period. Patients receiving any form of induction or adjuvant treatment (chemotherapy or radiation treatment) were excluded. The primary outcome measured was OS.

\section{Statistical analysis}

Data from the SEER database was imported on to the Statistical Package for the Social Sciences software (SPSS v.23.0, IBM Corp., USA) for further analysis. Unadjusted comparison between groups was conducted using the chi-square test or Fisher's exact test (as appropriate) for categorical variables and Student's $t$-test for continuous variables. Comparisons for non-parametric variables was done using Mann Whitney U test. Survival time was measured from the time of diagnosis to the time of death or last follow-up, median and 5-year survival indices were estimated by the Kaplan-Meier method and compared using log-rank test. We subsequently computed propensity scores to assess the probability of being assigned to a treatment group (segmentectomy or lobectomy), based on the observed patient's demographic, and pretreatment clinical characteristics. Logistic regression model was used to calculate the propensity scores derived from these confounding preoperative parameters. These variables were determined a priori and included age, sex, race, year of diagnosis, marital status and preoperative tumor characteristics (size, histology, tumor location). The most appropriately-matched pairs were selected based on the 
propensity scores using a 1:1 nearest neighbor algorithm (R-plugin 2.14.2 for SPSS v21.0). The standardized differences for each variable in the lobectomy and segmentectomy cohorts were 0.1 or less, except for tumor location, indicating well-matched samples. After the propensity score matching, differences in pre-treatment clinical and demographic variables between groups were further assessed using Pearson's chi-square or Fischer's exact test for categorical variables and Student's $t$-test for continuous variables. The Kaplan-Meier method was used to compare OS between the propensity matched groups. Additionally, multivariate cox-regression was used to study relationship between various patient and disease-specific factors and survival.

\section{Results}

We identified 591,071 patients with lung cancer between 2004 and 2015; 4,332 patients met our inclusion criteria. Lobectomy was done in 3,977 (91.8\%) patients while $355(8.2 \%)$ patients underwent segmentectomy. The median follow-up for the lobectomy group was 53 months whereas for the segmentectomy group it was 40 months. Baseline demographics and clinical characteristics for segmentectomy and lobectomy groups are detailed in Table 1. Patients undergoing lobectomy were found to be younger (mean: 67.4 vs. $70.2, \mathrm{P}<0.01$ ) and had a larger tumor size (mean: 15.3 vs. $14.7 \mathrm{~mm}, \mathrm{P}=0.04$ ).

\section{Survival analysis—unmatched cohort}

Patients who underwent lobectomy had a significantly higher OS than patients who underwent segmentectomy $(\mathrm{P}<0.01)$ (Figure 1). The median OS values was 90 and 62 months for lobectomy and segmentectomy respectively. One-, 3 - and 5 -year survival for the lobectomy group was $91.8 \%, 65.8 \%$ and $44.5 \%$, whereas for the segmentectomy group it was $90.7 \%, 56.9 \%$ and $32.1 \%$ respectively $(\mathrm{P}=0.46$, $\mathrm{P}<0.01$ and $\mathrm{P}<0.001$, respectively).

\section{Propensity score matching and multivariate survival analysis}

Propensity matching yielded 640 patients (320 matched pairs) from lobectomy and segmentectomy groups. There were no significant differences in baseline characteristics between the two groups after matching (Table 2). Patients following lobectomy had significantly higher OS when compared to patients undergoing segmentectomy $(\mathrm{P}=0.011)$ (Figure 2). The median OS values was 87 and 62 months for lobectomy and segmentectomy respectively. One-, 3- and 5 -year survival for the lobectomy group was $90.9 \%, 66.3 \%$ and $45.9 \%$ whereas for the segmentectomy group it was $91.3 \%, 57.5 \%$ and $33.8 \%$ respectively $(\mathrm{P}=0.89, \mathrm{P}<0.05$ and $\mathrm{P}<0.01)$. We further investigated the correlation between OS and other covariates in a Cox Regression Analysis. Multivariate analysis demonstrated that lobectomy was associated with significantly improved survival (HR: 0.84, 95\% CI: 0.714-0.989, $\mathrm{P}=0.036)$. Additionally, married status, adenocarcinoma histology and number of lymph nodes sampled were associated with better survival $(\mathrm{P}<0.05)$, while advanced age and male gender had worse survival outcomes $(\mathrm{P}<0.05)$ (Table 3).

\section{Discussion}

Since the results of the LCSG showed higher rate of recurrence and decreased OS with sublobar resection, lobectomy has remained the standard of care for early stage 1A NSCLC (4). Over the last two decades, a growing body of evidence that comprised primarily of large retrospective reviews, supported oncologic equivalence of segmentectomy and lobectomy for early stage NSCLC, and with better preservation of lung function (14,17-19). Previous studies have cited following criteria for segmentectomy: tumors $\leq 2 \mathrm{~cm}$, anatomically favorable location with margins $\geq 2 \mathrm{~cm}$, and no evidence of clinically positive lymph nodes (14). Further studies have explored the various tumor histopathological characteristics as a point of comparison between lobectomy and segmentectomy for early stage NSCLC (15). However, this is the first study to investigate the impact of highgrade tumors on OS in patients undergoing lobectomy or segmentectomy for $\leq 2 \mathrm{~cm}$ NSCLC.

Segmentectomy as compared to wedge resection has the distinct advantage of achieving oncologic outcomes comparable to lobectomy, in a select group of patients (14). However, caution must be exercised when selecting patients for segmentectomy purely on the grounds of tumor size and achievable margins $\geq 2 \mathrm{~cm}$. Certain histological types, such as large cell neuroendocrine tumor has been shown to exhibit significantly worse survival outcomes following wedge resection and segmentectomy, as compared to lobectomy $(14,15)$. Similarly, Eguchi et al. studied the 'spread through air spaces' (STAS) as a form of invasion, wherein tumor cells extend beyond the tumor edge within the lung parenchyma (20). Interestingly, patients with STAS who 
Table 1 Baseline demographics and clinical characteristics for patients with high grade (p)T1N0 ( $\leq 2 \mathrm{~cm})$ non-small cell lung cancer

\begin{tabular}{|c|c|c|c|c|}
\hline Parameters & Lobectomy $(n=3,977)$ & Segmentectomy $(n=355)$ & $P$ value & $\begin{array}{l}\text { Standardized } \\
\text { differences }\end{array}$ \\
\hline Age & & & $<0.01$ & 0.31 \\
\hline Mean/SEM & $67.4 / 0.143$ & $70.2 / 0.468$ & & \\
\hline Sex, n (\%) & & & 0.88 & 0.023 \\
\hline Race, n (\%) & & & 0.25 & 0.03 \\
\hline White & $3,484(87.6)$ & $316(89.0)$ & & \\
\hline Black & $294(7.4)$ & $28(7.9)$ & & \\
\hline Single & $427(10.7)$ & $39(11.0)$ & & \\
\hline Married & $2,232(56.1)$ & $184(51.8)$ & & \\
\hline Divorced & $540(13.6)$ & $45(12.7)$ & & \\
\hline Widowed & $587(14.8)$ & $71(20.0)$ & & \\
\hline Separated & $34(0.9)$ & $1(0.3)$ & & \\
\hline Unknown & $157(3.9)$ & $15(4.2)$ & & \\
\hline Tumor location, n (\%) & & & $<0.01$ & 0.18 \\
\hline Tumor histology, n (\%) & & & 0.18 & 0.03 \\
\hline Squamous cell carcinoma & $1,424(35.8)$ & $132(37.2)$ & & \\
\hline Adenocarcinoma & $1,887(47.4)$ & $160(45.1)$ & & \\
\hline Large cell carcinoma & $222(5.6)$ & $25(7.0)$ & & \\
\hline Neuroendocrine & $36(0.9)$ & $7(2.0)$ & & \\
\hline Other & $408(10.3)$ & $31(8.7)$ & & \\
\hline Tumor grade, $\mathrm{n}(\%)$ & & & 0.39 & 0.01 \\
\hline Poorly differentiated, grade III & $3,813(95.9)$ & $337(94.9)$ & & \\
\hline Undifferentiated, grade IV & $164(4.1)$ & $18(5.1)$ & & \\
\hline (p) T stage, n (\%) & & & $<0.01$ & \\
\hline (p)T1a & $530(13.3)$ & $66(18.6)$ & & \\
\hline (p)T1b & $3,447(86.7)$ & $289(81.4)$ & & \\
\hline
\end{tabular}

Table 1 (continued) 
Table 1 (continued)

\begin{tabular}{|c|c|c|c|c|}
\hline Parameters & Lobectomy $(n=3,977)$ & Segmentectomy $(n=355)$ & $P$ value & $\begin{array}{c}\text { Standardized } \\
\text { differences }\end{array}$ \\
\hline Tumor size (mm) & & & 0.04 & -0.17 \\
\hline Number of lymph nodes & & & $<0.01$ & \\
\hline
\end{tabular}

*, Standardized differences only presented for preoperative variables, used in propensity score analysis. SEM, standard error of the mean.

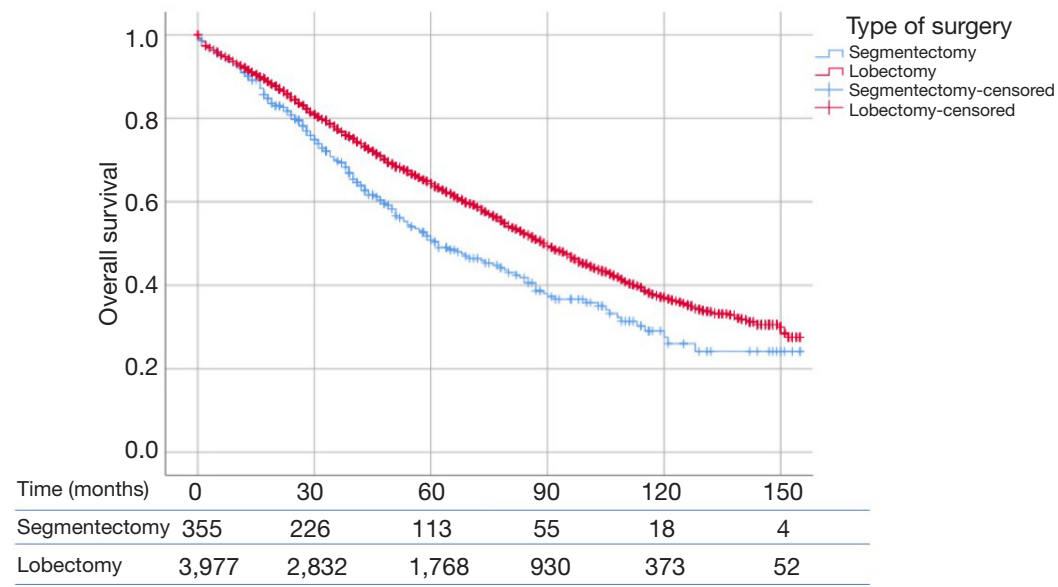

Figure 1 SEER database was used to study patients with high grade (p)T1N0M0 NSCLC ( $\leq 2 \mathrm{~cm}$ ), who underwent lobectomy or segmentectomy. OS was compared between lobectomy $(n=3,977)$ and segmentectomy $(n=355)$ using log-rank test. Patients who underwent lobectomy had a significantly longer OS than patients who underwent segmentectomy (90 vs. 62 months; P<0.01). SEER, Surveillance, Epidemiology and End Results; NSCLC, non-small cell lung cancer; OS, overall survival.

Table 2 Baseline demographics and clinical characteristics for high grade (p)T1N0 $(\leq 2 \mathrm{~cm})$ NSCLC and comparison between segmentectomy and lobectomy groups for propensity matched cohort

\begin{tabular}{lcc}
\hline Characteristics & Lobectomy & Segmentectomy \\
\hline Age, mean (years) & 68.75 & 69.25 \\
Sex, n (\%) & & 0.62 \\
Male & $133(41.6)$ & $156(48.8)$ \\
Female & $187(58.4)$ & $164(51.3)$ \\
Race, n (\%) & & $286(89.4)$ \\
White & $292(91.3)$ & $25(7.8)$ \\
Black & $15(4.7)$ & $7(2.2)$ \\
Asian & $10(3.1)$ & $2(0.6)$ \\
Other & $3(0.9)$ & 25 \\
\hline
\end{tabular}

Table 2 (continued) 
Table 2 (continued)

\begin{tabular}{|c|c|c|c|}
\hline Characteristics & Lobectomy & Segmentectomy & $P$ value \\
\hline Single & $24(7.5)$ & $35(10.9)$ & \\
\hline Married & $176(55.0)$ & $177(55.3)$ & \\
\hline Divorced & $44(13.8)$ & $42(13.1)$ & \\
\hline Separated & $3(0.9)$ & $1(0.3)$ & \\
\hline Unknown & $12(3.8)$ & $15(4.7)$ & \\
\hline Tumor histology, n (\%) & & & 0.66 \\
\hline Squamous cell carcinoma & $112(35)$ & $119(37.2)$ & \\
\hline Neuroendocrine & $2(0.6)$ & $5(1.6)$ & \\
\hline Other & $28(8.8)$ & $29(9.1)$ & \\
\hline Tumor grade, $\mathrm{n}(\%)$ & & & 0.56 \\
\hline Poorly differentiated, grade III & $308(96.3)$ & $305(95.3)$ & \\
\hline Undifferentiated, grade IV & $12(3.8)$ & $15(4.7)$ & \\
\hline (p)T stage, n (\%) & & & 0.83 \\
\hline (p)T1a & $47(14.7)$ & $49(15.3)$ & \\
\hline (p)T1b & $273(85.3)$ & $271(84.7)$ & \\
\hline
\end{tabular}

NSCLC, non-small cell lung cancer.

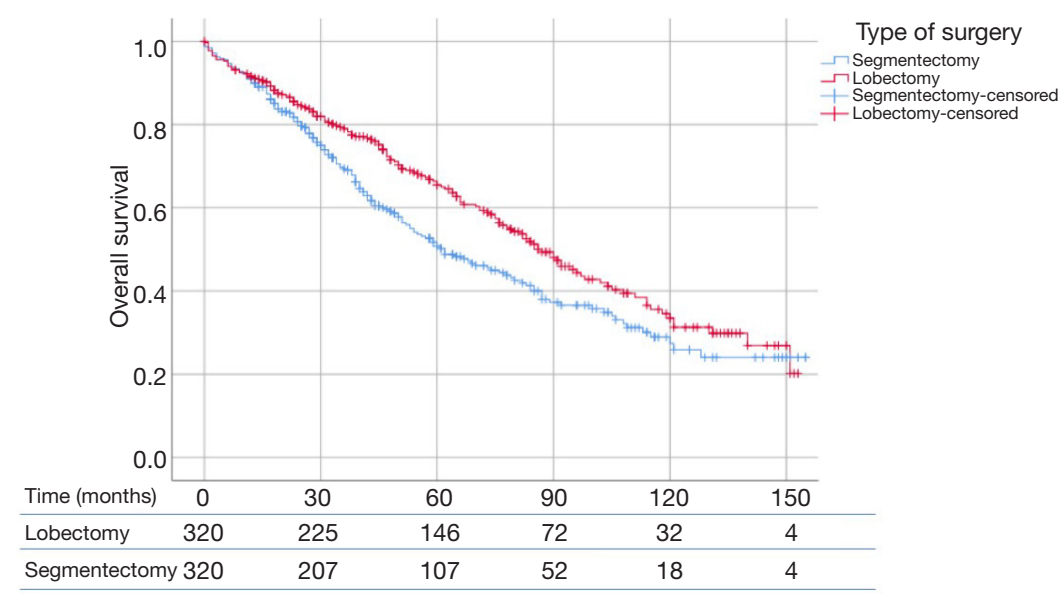

Figure 2 SEER database was used to study patients with high grade (p)T1N0M0 NSCLC ( $\leq 2 \mathrm{~cm}$ ), who underwent lobectomy or segmentectomy. Propensity score analysis was used to create 1:1 matched pairs between lobectomy and segmentectomy ( $\mathrm{n}=320$ each). Patients who underwent lobectomy had a significantly longer OS than patients who underwent segmentectomy (87 vs. 62 months; $\mathrm{P}=0.011)$. SEER, Surveillance, Epidemiology and End Results; NSCLC, non-small cell lung cancer; OS, overall survival. 
Table 3 Clinical and pathologic characteristics with associated HR based on cox regression multivariate analysis of OS in patients with high-grade $<2 \mathrm{~cm}$ NSCLC following lobectomy or segmentectomy

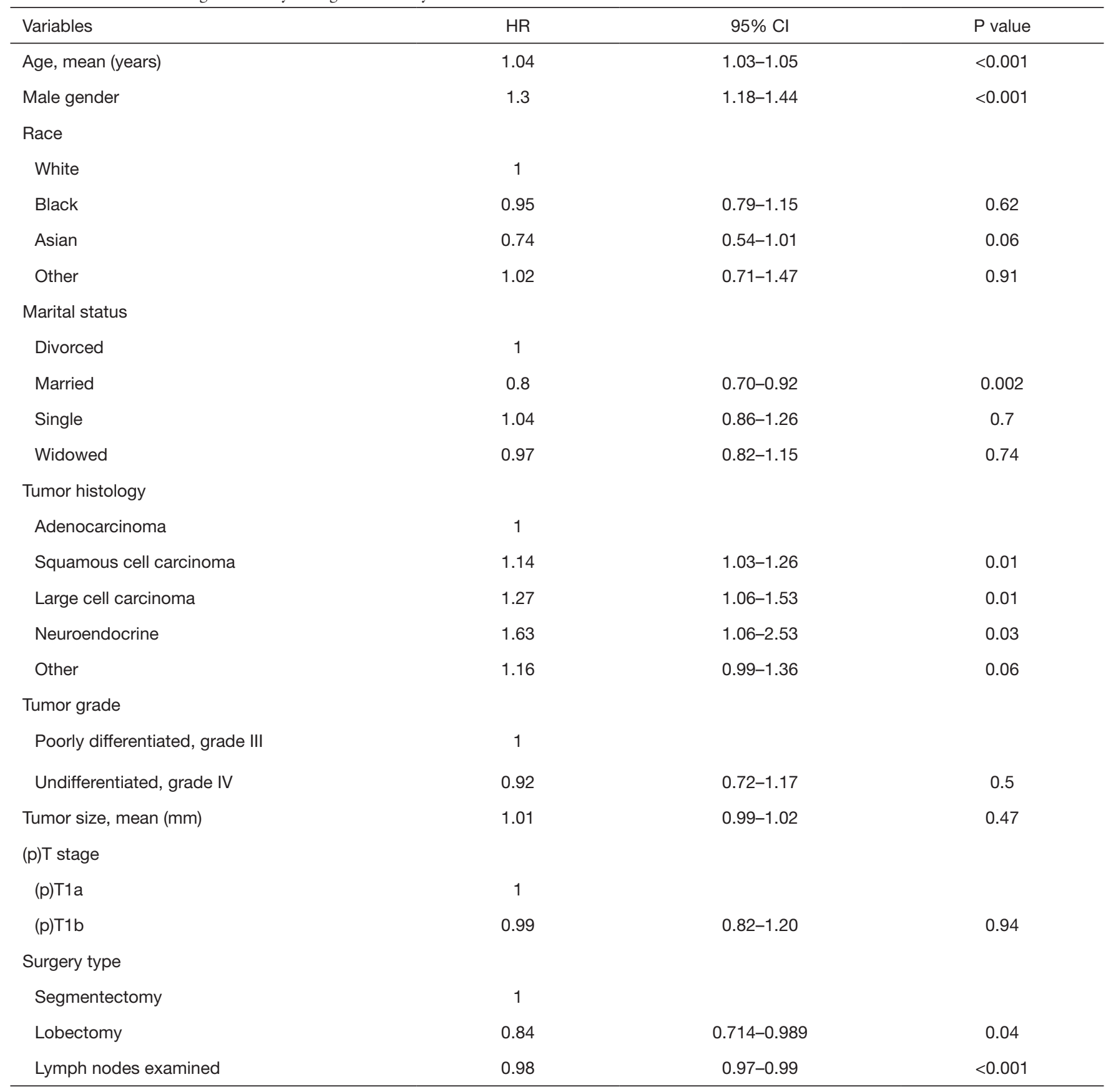

OS, overall survival; NSCLC, non-small cell lung cancer.

underwent sublobar resection as opposed to lobectomy for T1 NSCLC, showed higher risk of locoregional recurrence despite adequate margin to tumor ratio $(\geq 1)$. This was corroborated by Kim et al. and others, who have shown STAS and PD-L1 expression as important prognostic variables in patients with early stage NSCLC (21). On the contrary, the concept of tumor metabolic activity as measured by the PET scan [standard uptake value (SUV) $\max ]$ as a point of contention between lobectomy and segmentectomy was dispelled by Kamel et al., where the 
authors showed equivalent survival between lobectomy and segmentectomy for (c)T1N0 NSCLC, with SUV max values $\geq 3 \mathrm{~g} / \mathrm{dL}$ (22). Similarly, Razi et al. showed equivalent survival between lobectomy and segmentectomy for clinical stage IA lung cancer with unsuspected (p)N1/N2 disease, essentially challenging the dogma mandating lobectomy when unsuspected microscopic lymph node metastases is diagnosed intraoperatively (23). In essence, there are potentially host of yet unknown histopathological markers that may portend poorer prognosis with limited lung resection surgery.

Histologic grade of a tumor provides prognostic information in addition to that provided by the stage of the disease (24). A previous analysis of 5,018 hospital-based patients diagnosed between 1997 and 2003 and 712 population-based patients diagnosed between 1984 and 2003 with NSCLC revealed that histologic grade was significantly associated with survival after adjustment for the effects of age, sex, smoking status, tumor stage, histologic subtype and treatment administered (25). In our cohort of (p)stage 1, grade 3 and 4 NSCLC less than $2 \mathrm{~cm}$, we have shown that both unmatched and matched cohorts demonstrate significantly better survival outcomes for lobectomy as opposed to segmentectomy. To the best of our knowledge, our study is the first to compare segmentectomy and lobectomy specifically for high grade NSCLC and significantly enhances our understanding for appropriate patient selection for segmentectomy.

It is also important to appreciate that our study, being a population-based database is multi-institutional and mirrors the practice across the United States. However certain limitations exist to our data that is inherent to any large population-based study. Being a retrospective study, some degree of selection bias is inevitable. We tried to remedy this by using a propensity-matched and multivariate analysis. Secondly the SEER database does not contain information on patient comorbid conditions. These may be an important confounding factor when looking at survival outcomes as patients who undergo segmentectomy tend to have a greater number of comorbidities and reduced baseline lung function. Also important are the technical disparities among operators on what constitutes a segmentectomy. Another important distinction that could not be made from SEER database, is whether the information on histologic grade was available prior to planned operation, or on final pathology after the surgical resection.

\section{Conclusions}

Based on our analysis of 4,332 patients over 12 years, we conclude that lobectomy is associated with better longterm survival outcomes as compared to segmentectomy for small peripheral NSCLC $\leq 2 \mathrm{~cm}$ and high grades of tumor differentiation. We believe that additional work is needed to further refine the criteria for segmentectomy, stratifying various NSCLC histologies with their respective grades to allow for more comprehensive selection for segmentectomy.

\section{Acknowledgments}

Funding: None.

\section{Footnote}

Reporting Checklist: The authors have completed the STROBE reporting checklist. Available at http://dx.doi. org/10.21037/jtd-20-1530

Peer Review File: Available at http://dx.doi.org/10.21037/jtd20-1530

Conflicts of Interest: All authors have completed the ICMJE uniform disclosure form (available at http://dx.doi. org/10.21037/jtd-20-1530). The authors have no conflicts of interest to declare.

Ethical Statement: The authors are accountable for all aspects of the work in ensuring that questions related to the accuracy or integrity of any part of the work are appropriately investigated and resolved. Our study conforms to the Declaration of Helsinki (as revised in 2013). Since our study utilizes de-identified data from a publicly accessible database, individual consent is waived and our study is exempt from IRB review.

Open Access Statement: This is an Open Access article distributed in accordance with the Creative Commons Attribution-NonCommercial-NoDerivs 4.0 International License (CC BY-NC-ND 4.0), which permits the noncommercial replication and distribution of the article with the strict proviso that no changes or edits are made and the original work is properly cited (including links to both the formal publication through the relevant DOI and the license). See: https://creativecommons.org/licenses/by-nc-nd/4.0/.

\section{References}

1. Tanoue LT, Tanner NT, Gould MK, et al. Lung cancer 
screening. Am J Respir Crit Care Med 2015;191:19-33.

2. Zhao M, Lu T, Huang Y, et al. Survival and long-term cause-specific mortality associated with stage IA lung adenocarcinoma after wedge resection vs. segmentectomy: a population-based propensity score matching and competing risk analysis. Front Oncol 2019;9:593.

3. Lim TY, Park S, Kang CH. A meta-analysis comparing lobectomy versus segmentectomy in stage I non-small cell lung cancer. Korean J Thorac Cardiovasc Surg 2019;52:195-204.

4. Cao J, Yuan P, Wang Y, et al. Survival rates after lobectomy, segmentectomy, and wedge resection for non-small cell lung cancer. Ann Thorac Surg 2018;105:1483-91.

5. Zhong C, Sakurai H, Wei S, et al. Sublobar resections for small-sized stage Ia lung adenocarcinoma: a Sino-Japanese multicenter study. J Thorac Dis 2018;10:991-8.

6. Tanvetyanon T, Keenan R. Recovery of lung function after segmentectomy versus lobectomy for early-stage lung cancer. J Thorac Dis 2018;10:S2144-6.

7. Zhao ZR, Situ DR, Lau RWH, et al. Comparison of segmentectomy and lobectomy in stage IA adenocarcinomas. J Thorac Oncol 2017;12:890-6.

8. Ijsseldijk MA, Shoni M, Siegert C, et al. Oncological outcomes of lobar resection, segmentectomy, and wedge resection for T1a non-small-cell lung carcinoma: a systematic review and meta-analysis. Semin Thorac Cardiovasc Surg 2020;32:582-90.

9. Whitson BA, Groth SS, Andrade RS, et al. Survival after lobectomy versus segmentectomy for stage I non-small cell lung cancer: a population-based analysis. Ann Thorac Surg 2011;92:1943-50.

10. Abbas AE. Surgical management of lung cancer: history, evolution, and modern advances. Curr Oncol Rep 2018;20:98.

11. Charloux A, Quoix E. Lung segmentectomy: does it offer a real functional benefit over lobectomy? Eur Respir Rev 2017;26:170079.

12. Tane S, Nishio W, Nishioka $Y$, et al. Evaluation of the residual lung function after thoracoscopic segmentectomy compared with lobectomy. Ann Thorac Surg 2019;108:1543-50.

13. Moon MH, Moon YK, Moon SW. Segmentectomy versus lobectomy in early non-small cell lung cancer of 2 cm or less in size: a population-based study. Respirology 2018;23:695-703.

14. Okumura M, Goto M, Ideguchi K, et al. Factors associated with outcome of segmentectomy for non-small cell lung cancer: long-term follow-up study at a single institution in
Japan. Lung Cancer 2007;58:231-7.

15. Lutfi $W$, Schuchert MJ, Dhupar R, et al. Sublobar resection is associated with decreased survival for patients with early stage large-cell neuroendocrine carcinoma of the lung. Interact Cardiovasc Thorac Surg 2019;29:517-24.

16. Surveillance Research Program, National Cancer Institute SEER*Stat software. version 8.3.6r. Available online: https://seer.cancer.gov/seerstat/

17. Echavarria MF, Cheng AM, Velez-Cubian FO, et al. Comparison of pulmonary function tests and perioperative outcomes after robotic-assisted pulmonary lobectomy vs segmentectomy. Am J Surg 2016;212:1175-82.

18. Dziedzic R, Żurek W, Marjański T, et al. Stage I nonsmall-cell lung cancer: long-term results of lobectomy versus sublobar resection from the Polish National Lung Cancer Registry†. Eur J Cardiothorac Surg 2017;52:363-9.

19. Bedetti B, Bertolaccini L, Rocco R, et al. Segmentectomy versus lobectomy for stage I non-small cell lung cancer: a systematic review and meta-analysis. J Thorac Dis 2017;9:1615-23.

20. Eguchi T, Kameda K, Lu S, et al. Lobectomy is associated with better outcomes than sublobar resection in spread through air spaces (STAS)-positive T1 lung adenocarcinoma: a propensity score-matched analysis. J Thorac Oncol 2019;14:87-98.

21. Kim M, Chung Y, Kim K, et al. Prognostic factors of acinar- or papillary-predominant adenocarcinoma of the lung. Lung Cancer 2019;137:129-35.

22. Kamel MK, Rahouma M, Lee B, et al. Segmentectomy is equivalent to lobectomy in hypermetabolic clinical stage IA lung adenocarcinomas. Ann Thorac Surg 2019;107:217-23.

23. Razi SS, Nguyen D, Villamizar N. Lobectomy does not confer survival advantage over segmentectomy for nonsmall cell lung cancer with unsuspected nodal disease. J Thorac Cardiovasc Surg 2020;159:2469-83.e4.

24. Carriaga MT, Henson DE. The histologic grading of cancer. Cancer 1995;75:406-21.

25. Sun Z, Aubry M, Deschamps C, et al. Histologic grade is an independent prognostic factor for survival in nonsmall cell lung cancer: an analysis of 5018 hospital- and 712 population-based cases. J Thorac Cardiovasc Surg 2006;131:1014-20.

Cite this article as: Baig MZ, Razi SS, Weber JF, Connery CP, Bhora FY. Lobectomy is superior to segmentectomy for peripheral high grade non-small cell lung cancer $\leq 2 \mathrm{~cm}$. J Thorac Dis 2020;12(10):5925-5933. doi: 10.21037/jtd-20-1530 